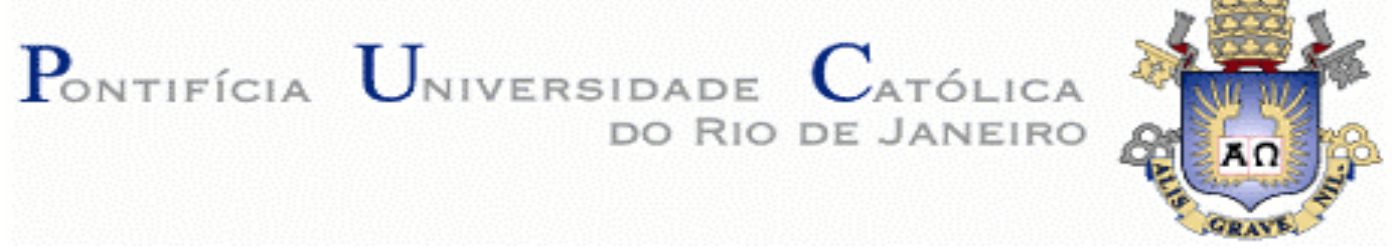

Jorge Kenedy Almeida Silva

Regulamentação técnica: proposição de um código mínimo das boas práticas e diagnóstico do sistema brasileiro.

Dissertação de Mestrado

Dissertação apresentada como requisito parcial para obtenção do título de Mestre pelo Programa de PósGraduação em Metrologia da PUC-Rio. Área de concentração: Metrologia para Qualidade e Inovação.

Orientador: Mauricio Nogueira Frota, Ph.D. 


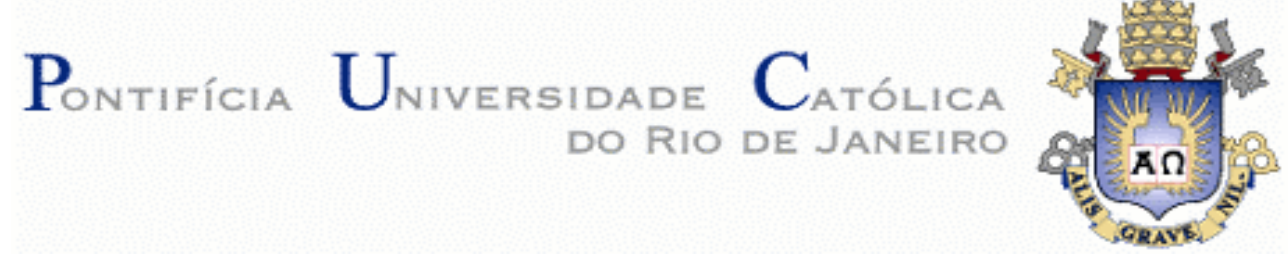

Jorge Kenedy Almeida Silva

\title{
Regulamentação técnica: proposição de um código mínimo das boas práticas e diagnóstico do sistema brasileiro.
}

\begin{abstract}
Dissertação apresentada como requisito parcial para obtenção do título de Mestre pelo Programa de PósGraduação em Metrologia, Qualidade e Inovação do Centro Técnico Científico da Pontifícia Universidade Católica do Rio de Janeiro. Aprovada pela Comissão Examinadora e homologada pela Coordenação Setorial de Pós-Graduação, formalizado pelas respectivas assinaturas.
\end{abstract}

Comissão Examinadora:

Prof. Mauricio Nogueira Frota, Ph.D. Orientador

Programa de Pós-graduação em Metrologia (PósMQI) Pontifícia Universidade Católica do Rio de Janeiro (PUC-Rio)

Profa. Elisabeth Costa Monteiro, Dra.

Programa de Pós-Graduação em Metrologia (PósMQI) Pontifícia Universidade Católica do Rio de Janeiro (PUC-Rio)

Prof. Antônio José Junqueira Botelho, Ph.D.

Programa de Pós-Graduação em Metrologia (PósMQI) Pontifícia Universidade Católica do Rio de Janeiro (PUC-Rio)

Profa. Maria de Fátima Ludovico de Almeida, Dra. Petrobras Transporte S.A. (Transpetro)

Coordenação Setorial de Pós-Graduacão:

Prof. José Eugênio Leal, Dr. Coordenador Setorial de Pós-Graduação do Centro Técnico Científico PUC-Rio

Rio de Janeiro, 14 de fevereiro de 2007. 
Todos os direitos reservados. É proibida a reprodução total ou parcial do trabalho sem autorização da universidade, do autor e do orientador.

\section{Jorge Kenedy Almeida Silva}

Graduado em Administração de Empresas (1992) e Matemática (2002), Especialização em Matemática (2004) pela Universidade do Grande Rio (Unigranrio). Oficial do Exército Brasileiro, no posto de $1^{0}$ Tenente de $2^{\mathrm{a}}$ Classe, da Arma de Infantaria (1989-93).

Ficha Catalográfica

Silva, Jorge Kenedy Almeida
Regulamentação técnica: proposição de um código
mínimo das boas práticas e diagnóstico do sistema
brasileiro / Jorge Kenedy Almeida Silva; orientador:
Mauricio Nogueira Frota-2007.
$185 \mathrm{f}$ : : il. ; $30 \mathrm{~cm}$.
Dissertação (Mestrado em Metrologia)-Pontifícia
Universidade Católica do Rio de Janeiro, Rio de Janeiro,
2007 .
Inclui bibliografia
1. Metrologia. 2. Normas técnicas. 3. Regulamentação
técnica. 4. Boas práticas da regulamentação.

CDD: 389.1 
À minha esposa Simone Simões de Oliveira Silva e filha Michele Simões Almeida Silva, pelo apoio, carinho e confiança. 


\section{Agradecimentos}

Ao orientador Professor Doutor Maurício Nogueira Frota, coordenador do Programa de Pós-Graduação em Metrologia da PUC-Rio, pelo apoio, dedicada orientação presente em todos os momentos de concepção, reflexão e desenvolvimento da presente dissertação de mestrado, registrando-se, a confiança depositada e o permanente estímulo ao meu desenvolvimento profissional.

Ao Mestre Pedro Paulo Almeida Silva, Assessor da Diretoria de Metrologia Legal do Inmetro, pelo mais explícito estímulo para realização desta dissertação.

Aos profissionais do Instituto Nacional de Metrologia, Normalização e Qualidade Industrial (Inmetro), pelas informações fornecidas relacionadas à regulamentação técnica brasileira, destacando-se a desprendida colaboração de Î́ris Trindade Chacon (Chefe da Divisão de Instrumentos de Massa Específica, Temperatura e Outros), Mauricio Martinelli Réche (Chefe da Divisão de Desenvolvimento e Regulamentação Metrológica da Diretoria de Metrologia Legal), Anna Camboim (Gerente do Ponto Focal de Barreiras Técnicas às Exportações - Inmetro).

Aos órgãos regulamentadores brasileiros que colaboraram com o desenvolvimento do trabalho aceitando responder o instrumento de coleta de dados.

À PUC-Rio, pelo ambiente acadêmico-científico favorável no desenvolvimento deste trabalho e ao CNPq, pela bolsa concedida.

Ao especialista da Qualidade Marcus Vinicius Porto, pela dedicada contribuição nas etapas de definição do tema e reflexões sobre o instrumento de coleta de dados.

À secretária do Mestrado em Metrologia para Qualidade e Inovação da PUC-Rio, Márcia Ribeiro Teixeira, pelo permanente suporte administrativo e presteza com que sempre atende nossas demandas.

Aos meus colegas e companheiros do Pós MQI/PUC-Rio, por colaborarem na manutenção do ambiente criativo e agradável que juntos compartilhamos no acolhedor campus da PUC-Rio, ambiente de estímulo e inovação.

Aos professores pelos ensinamentos e aos funcionários pelo desprendido suporte.

Aos professores que aceitaram participar da comissão examinadora pelas relevantes sugestões.

Aos amigos Jaime Mamani Ticona, Ricardo Antunes Gomes, Arnaldo Melgaço da Silva, Márcio Cândido da Silva e Sérgio Henrique Silva Júnior, sempre presentes nessa fascinante caminhada.

Aos amigos e professores Lindonor G. Siqueira, Ângelo S. Siqueira, Jéferson Dias dos Santos, Jonas Luiz Pedroza e Lenir de Pina Jordão, pelo estímulo constante.

A minha esposa Simone Simões de Oliveira Silva e a minha filha Michele Simões Almeida Silva que participaram diretamente do processo de motivação.

Aos meus pais, irmãos e sobrinhos pelo carinho e estímulo.

E também, a todos aqueles que direta ou indiretamente colaboraram para o desenvolvimento do presente trabalho. 


\section{Resumo}

Silva, Jorge kenedy Almeida; Frota, Maurício Nogueira. Regulamentação técnica: proposição de um código mínimo das boas práticas e diagnóstico do sistema brasileiro. Rio de Janeiro, 2007. 185 p. Dissertação de Mestrado - Programa de Pós-Graduação em Metrologia. Área de concentração: Metrologia para Qualidade e Inovação (Pós-MQI), Pontifícia Universidade Católica do Rio de Janeiro.

Objetivo: Proposição de um código mínimo das 'boas práticas da regulamentação técnica’ com vistas a disciplinar o desenvolvimento e a aplicação de regulamentos e assegurar que estes somente sejam introduzidos quando justificados com base em objetivos legítimos e em benefício da eficiência de qualquer sistema regulatório, com o propósito de evitar barreiras desnecessárias ao comércio e sem prejuízo dos interesses públicos. Motivação: Contribuir para o avanço do conhecimento sobre regulamentação técnica e para o aprimoramento das práticas regulatórias do sistema brasileiro, assim facilitando a inserção comercial do País em âmbito internacional. Contextualização: O trabalho se desenvolveu no contexto de um movimento mundial de harmonização de nomenclaturas e procedimentos regulatórios ainda em uso nos países membros da Organização Mundial do Comércio, reconhecendo-se, entretanto, que aspectos puramente técnicos não são capazes de justificar um modelo unificado. Metodologia: A proposição das boas práticas da regulamentação baseou-se: (i) na análise das características mínimas dos sistemas regulatórios em relação às recomendações da Organização Mundial do Comércio, do Código de Alfândega e de acordos comerciais regionais e internacionais de conceituados organismos; (ii) no diagnóstico da regulamentação técnica vigente no País à luz da prática internacional e na identificação dos diferentes atores que integram o sistema regulatório brasileiro; e (iii) na identificação dos aspectos funcionais e na eficácia do sistema regulatório brasileiro, por meio de pesquisa de campo conduzida junto aos 29 organismos que integram o referido sistema. Resultados: À luz da prática internacional, o trabalho identifica, discute e fundamenta um conjunto de nove parâmetros considerados essenciais às boas práticas da regulamentação, a saber: (i) aderência aos preceitos básicos e nomenclatura internacional; (ii) coordenação integrada do sistema nacional de regulamentação; (iii) sistemática para avaliação de riscos pela introdução ou não de um determinado regulamento técnico; (iv) infra-estrutura de comitês técnicos; (v) sistema nacional de normalização; (vi) sistema nacional de metrologia; (vii) infra-estrutura básica para avaliação da conformidade; (viii) redes de segurança do sistema regulatório e (ix) acompanhamento de mercado. Conclusões: A introdução de um código de boas práticas da regulamentação constitui pré-condição ao adequado funcionamento de qualquer sistema regulatório. Nove atributos essenciais foram caracterizados à luz da prática internacional como elementos que devem integrar um código mínimo capaz de proporcionar a harmonização entre os órgãos regulamentadores do país, a interação dos órgãos regulamentadores com o Ponto Focal Brasileiro, a promoção da cidadania, o desenvolvimento econômico e a redução dos impactos sócio-econômico-ambientalcultural da regulamentação técnica.

\section{Palavras-chave:}

1. Metrologia. 2. Normas técnicas. 3. Regulamentação técnica. 4. Boas práticas da regulamentação. 


\section{Abstract}

Silva, Jorge Kenedy Almeida; Frota, Maurício Nogueira. Technical regulation: proposition of a minimum code of the good regulatory practices and diagnosis of the Brazilian system. Rio de Janeiro, 2007. 185p. Master dissertation. - Posgraduation programme in Metrology. Area of concentration: Metrology for quality and innovation. Pontifical Catholic University of Rio de Janeiro (PUC-Rio).

Objectives: The aim of this study is to formalize a set of rules, principles and instruments to suit the purpose of a "good technical regulatory practice" that would discipline the development and application of technical regulations based on legitimate objectives oriented by public interests. Such framework is required for the benefit of an efficient regulatory system to avoid unnecessary barriers to trade and without loss of public interests. Motivation: To contribute to the advance of technical regulation and best regulatory practices in Brazil, as a strategy to promote the country internationally. Context: The study was developed in the context of a worldwide movement, coordinated by the WTO, to harmonize nomenclature and procedures and to develop good regulatory practices, recognizing that purely technical aspects are not suffice to justify an unified approach. Methodology: The good regulatory practice here proposed is based on: (i) a diagnosis of technical regulation in place (ii) a search of the minimum characteristics of the regulatory systems in the light of WTO recommendations and relevant commercial agreements and (iii) a review of the specialized literaure and an exploratory survey among the regulatory bodies to find out their functional and operational aspects. Results: In the light of international practices, this work identifies, discusses and establishes a set of guiding principles considered essential to good regulatory practice, namely: (i) adherence to basic precepts and international practices; (ii) risk assessment associated with the introduction or not of a specific regulation; (iii) integrated coordination of a national system of regulation; (iv) infra-structure of national committees; (v) a national system of standardization; (vi) a national system of metrology; (vii) basic infra-structure for conformity assessment; (viii) security networks for the regulatory system and (ix) market surveillance. Conclusions: The introduction of a code of regulatory practices constitutes a pre-condition to an adequate functioning of any regulatory system. In the light of the best international regulatory practices, nine essential attributes were identified to promote harmonization of the regulatory bodies and information exchange between them; citizenship; economic development and reduction of their cultural, environmental, economic and social impacts.

Keywords: 1. Metrology. 2. Technical standards. 3. Technical regulation. 4. Good regulatory practice. 


\section{Sumário}

10 ambiente do sistema regulatório 16

1.1. Agências reguladoras: perspectiva histórica 17

1.2. Regulação e regulamentação, uma dualidade de conceito 22

1.3. Objetivo, motivação e contexto 23

1.4. Metodologia $\quad 24$

1.5. Estrutura do trabalho 25

2 Fundamentos da regulamentação técnica 27

2.1. Considerações sobre o Acordo TBT 28

2.2. Orientações para a União Européia: uma abordagem robusta 30

2.3. Orientações para ação alfandegária 34

2.4. Norma e regulamento técnico: conceituação 34

2.4.1. Inter-relacionamento entre os conceitos 37

2.4.2. Regulamentação: instrumento de redução de barreiras técnicas 38

2.4.3. Normalização: acesso a fontes de informação 40

2.4.4. Regulamentação: acesso a fontes de informação 41

2.5. A regulamentação técnica nos países: diferentes abordagens 42

2.6. Principais organismos internacionais de normalização 43

2.7. Preceitos básicos da harmonização 45

2.8. Normas internacionais: uma estratégia de harmonização 46

2.9. A importância da gestão da qualidade na regulamentação 47

2.10. Requisitos impostos sobre embalagens no comércio 50

2.10.1. Etiquetagem 51

2.10.2. Eco-etiquetagem 52

3 As boas práticas da regulamentação técnica 55

3.1. Aderência aos preceitos básicos $\quad 59$

3.1.1. Taxonomia da regulamentação 60

3.1.2. Adequação da resposta regulatória 61

3.2. Sistema nacional de regulamentação: coordenação 62

3.2.1. Comissão nacional para assuntos regulatórios 62

3.2.2. Negociações de comércio: resultados esperados 64

3.3. Sistemática de avaliação de riscos $\quad 67$

3.4. O sistema nacional de normalização $\quad 71$

3.5. O sistema nacional de metrologia $\quad 72$

3.5.1. Metrologia científica $\quad 72$

3.5.2. Metrologia industrial $\quad 73$

$\begin{array}{ll}\text { 3.5.3. Metrologia legal } & 73\end{array}$ 
3.6. Infra-estrutura de comitês técnicos $\quad 79$

3.7. Infra-estrutura básica para avaliação da conformidade 80

3.7.1. Mecanismos $\quad 81$

3.7.2. Classificação $\quad 85$

3.7.3. Natureza $\quad 85$

3.8. Redes de segurança do sistema regulatório 85

3.8.1. Redes de segurança baseada em estatutos 86

3.8.2. Sistema de leis civis 86

3.9. Acompanhamento de mercado $\quad 86$

3.9.1. Atividades de fiscalização 88

3.9.2. Monitoração de produtos introduzidos no mercado 88

3.9.3. Ações corretivas $\quad 89$

3.9.4. Atividades complementares $\quad 89$

3.10. Considerações sobre um código mínimo 90

4 Análise do sistema regulatório brasileiro $\quad 94$

4.1. Vertente de análise 1: acervo documental e institucional 95

4.1.1. Pesquisa do acervo documental 95

4.1.2. Pesquisa do acervo institucional (órgãos regulamentadores) 97

4.2. Vertente de análise 2: elementos essenciais do sistema regulatório 103

4.2.1. Aderência aos preceitos básicos 103

4.2.2. Coordenação integrada do sistema de regulamentação 104

4.2.3. Sistemática de avaliação de riscos 106

4.2.4. Sistema Brasileiro de Normalização (SBN) 106

$\begin{array}{ll}\text { 4.2.5. Sistema Nacional de Metrologia } & 108\end{array}$

4.2.6. Infra-estrutura de comitês técnicos 110

4.2.7. Infra-estrutura brasileira para avaliação da conformidade 113

4.2.8. Redes de segurança do sistema regulatório brasileiro 115

4.2.9. Ações de acompanhamento de mercado no Brasil 116

4.2.10. Considerações sobre a necessidade de um código mínimo no Brasil 121

4.3. Vertente de análise 3: análise da eficácia do sistema regulatório 122

4.3.1. Formulação da pesquisa de campo 122

4.3.2. Consistência da pesquisa de campo 125

5 Conclusões e recomendações $\quad 127$

Recomendações 132

$\begin{array}{ll}\text { Referências bibliográficas } & 134\end{array}$

Apêndice A. Instrumento de coleta de dados: elementos da regulamentação técnica brasileira 
Apêndice B. Especialistas participantes 150

Apêndice C. Processamento dos dados coletados 151

Anexo 1. Lei $\mathrm{n}^{\circ}$. 5.966, dezembro 11, 1973

Anexo 2. Lei $n^{\circ}$. 9.933, dezembro 20, 1999

Anexo 3. Resolução $n^{\circ}$. 11/75 162

Anexo 4. Resolução $n^{\circ}$. 02, janeiro 8, 1992

Anexo 5. Resolução $\mathrm{n}^{\circ}$. 06, agosto 24, 1992

Anexo 6. Resolução $\mathrm{n}^{\circ}$. 04, maio 26, 1993

Anexo 7. Resolução $n^{\circ}$. 01, maio 19, 1995

Anexo 8. Resolução $n^{\circ}$. 05, setembro 4, 1995

Anexo 9. Resolução $\mathrm{n}^{\circ}$. 02, junho 9, 2005

Anexo 10. Addendum (Instrumento do Ponto Focal) 179

Anexo 11. Corrigendum (Instrumento do Ponto Focal) 180

Anexo 12. Notification (Instrumento do Ponto Focal) 181

Anexo 13. Revision (Instrumento do Ponto Focal) 183

Anexo 14. Communication from Brazil (Ponto Focal) 184 


\section{Lista de Figuras}

Figura 1. Modelo de sistema de gestão da qualidade baseado em processo ......48

Figura 2. Etapas fundamentais da análise de impacto de risco (RIA)..................68

Figura 3. Plano de Regulamentação: Matriz Risco-Probabilidade....................... 69

Figura 4. Funções das boas práticas da regulamentação técnica..........................91

Figura 5. Estruturas, níveis hierárquicos e modelo para implementação de um

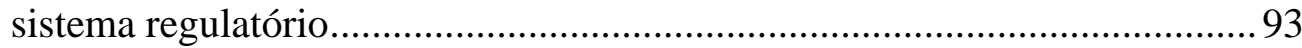

\section{Lista de Tabelas}

Tabela 1. Exemplos de eco-etiquetagem no âmbito de países.............................. 53

Tabela 2. Exemplos de rótulos ambientais regionais........................................... 54

Tabela 3. Exemplos de rótulos ambientais internacionais.................................... 54

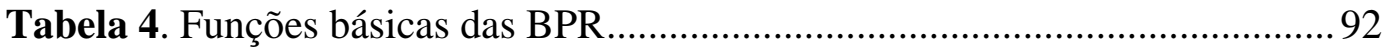




\section{Abreviaturas e siglas utilizadas}

Abimaq _ - Associação Brasileira da Indústria de Máquinas e Equipamentos

Abinee — - Associação Brasileira da Indústria Elétrica e Eletrônica

ABNT $\quad-$ Associação Brasileira de Normas Técnicas

AMN $\quad-$ Acordo Mercosul de Normalização

ANA $\quad-\quad$ Agência Nacional de Águas

Aneel _ - Agência Nacional de Energia Elétrica

Anatel _ - Agência Nacional de Telecomunicações

Antaq _ - Agência Nacional de Transportes Aquaviários

ANTT $\quad-$ Agência Nacional de Transportes Terrestres

Anvisa $\quad$ - Agência Nacional de Vigilância Sanitária

Apec $\quad-$ Asian Pacific Economic Co-operation

ANP $\quad-\quad$ Agência Nacional do Petróleo

ANS $\quad-$ Agência Nacional de Saúde

Aplac $\quad-$ Asia Pacific Laboratory Accreditation Co-operation

Arso $\quad-$ African Regional Organization for Standardization

BIPM $\quad-$ Bureau International des Poids et Mesures

BPR — - Boas Práticas da Regulamentação

CAC $\quad-$ Codex Alimentarius Commission

Cade

Caint

Can

Casco

CBAC

CBTC

CBM

CBN

CBR

CCAB

CEN

- Conselho Administrativo de Direito Econômico

- Coordenação Geral de Articulação Internacional

- Comunidade Andina

- Committee on Conformity Assessment

- Comitê Brasileiro de Avaliação da Conformidade

- Comitê de Coordenação de Barreiras Técnicas ao Comércio

- Comitê Brasileiro de Metrologia

- Comitê Brasileiro de Normalização

- Comitê Brasileiro de Regulamentação

- Comitê Codex Alimentarius do Brasil

Cenelec $\quad-$ European Committee for Electrotechnical Standardization

CGQV — - Coordenação Geral de Qualidade Vegetal

Cnen $\quad-\quad$ Comissão Nacional de Energia Nuclear

CNI $\quad-$ Confederação Nacional da Indústria

Conama _ - Conselho Nacional do Meio Ambiente

Conmetro - Conselho Nacional de Metrologia, Normalização e Qualidade Industrial

Copant $\quad-$ Comissão Pan-Americana de Normas Técnicas

CPCON - Comissão Permanente de Consumidores

CTA $\quad-$ Centro Tecnológico Aeroespacial

Ctex _ - Centro Tecnológico do Exército

Denatran - Departamento Nacional de Trânsito

DPC $\quad-$ Diretoria de Portos e Costas

DPDC — - Departamento de Proteção e Defesa do Consumidor

DNPM _ - Departamento Nacional de Produção Mineral

EA $\quad-$ European Co-operation for Accreditation

EFTA $\quad-$ European Fair Trade Association

ETSI $\quad-$ European Telecommunication Standards Institute

FAO $\quad-$ Food and Agriculture Organization of the United Nations 


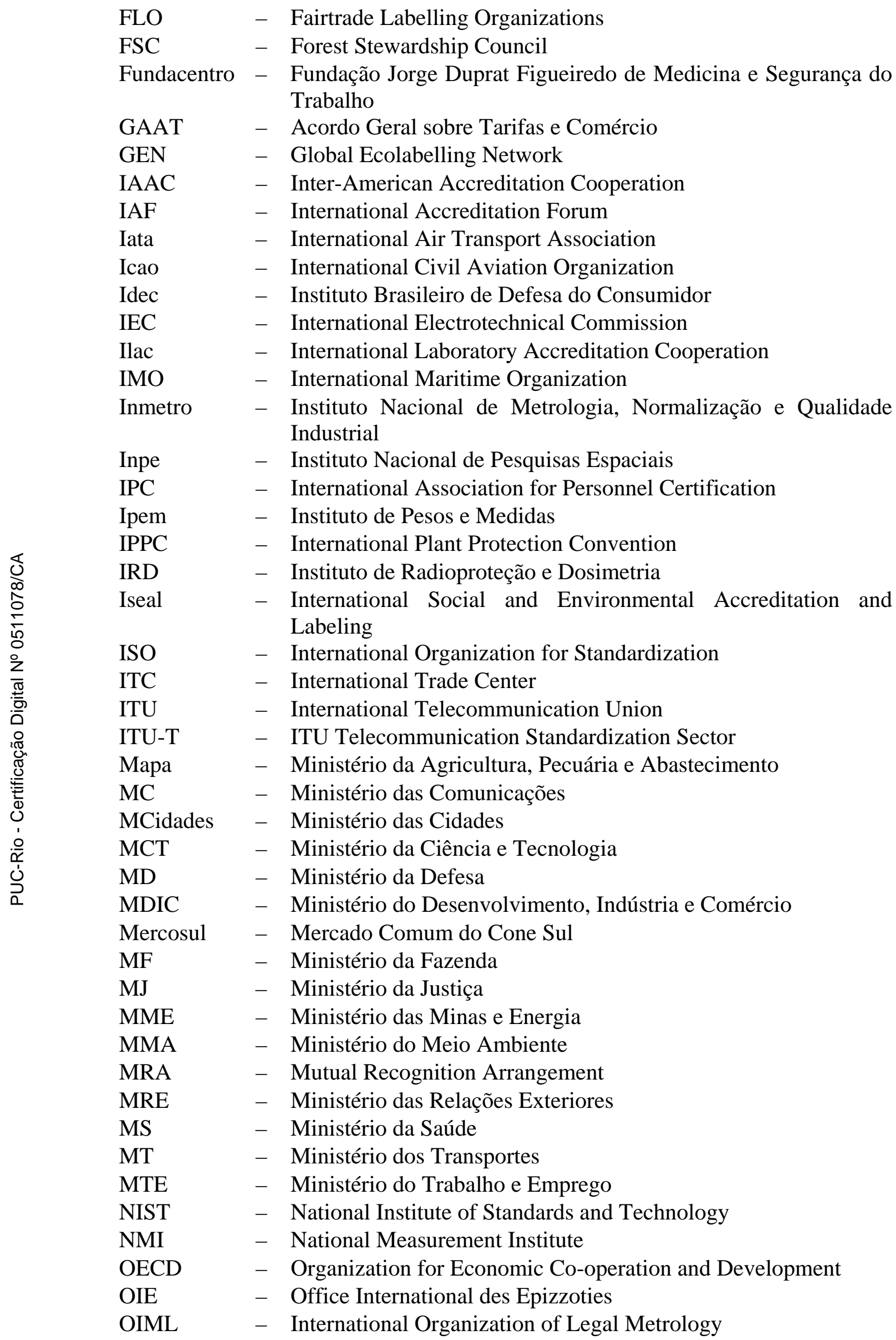




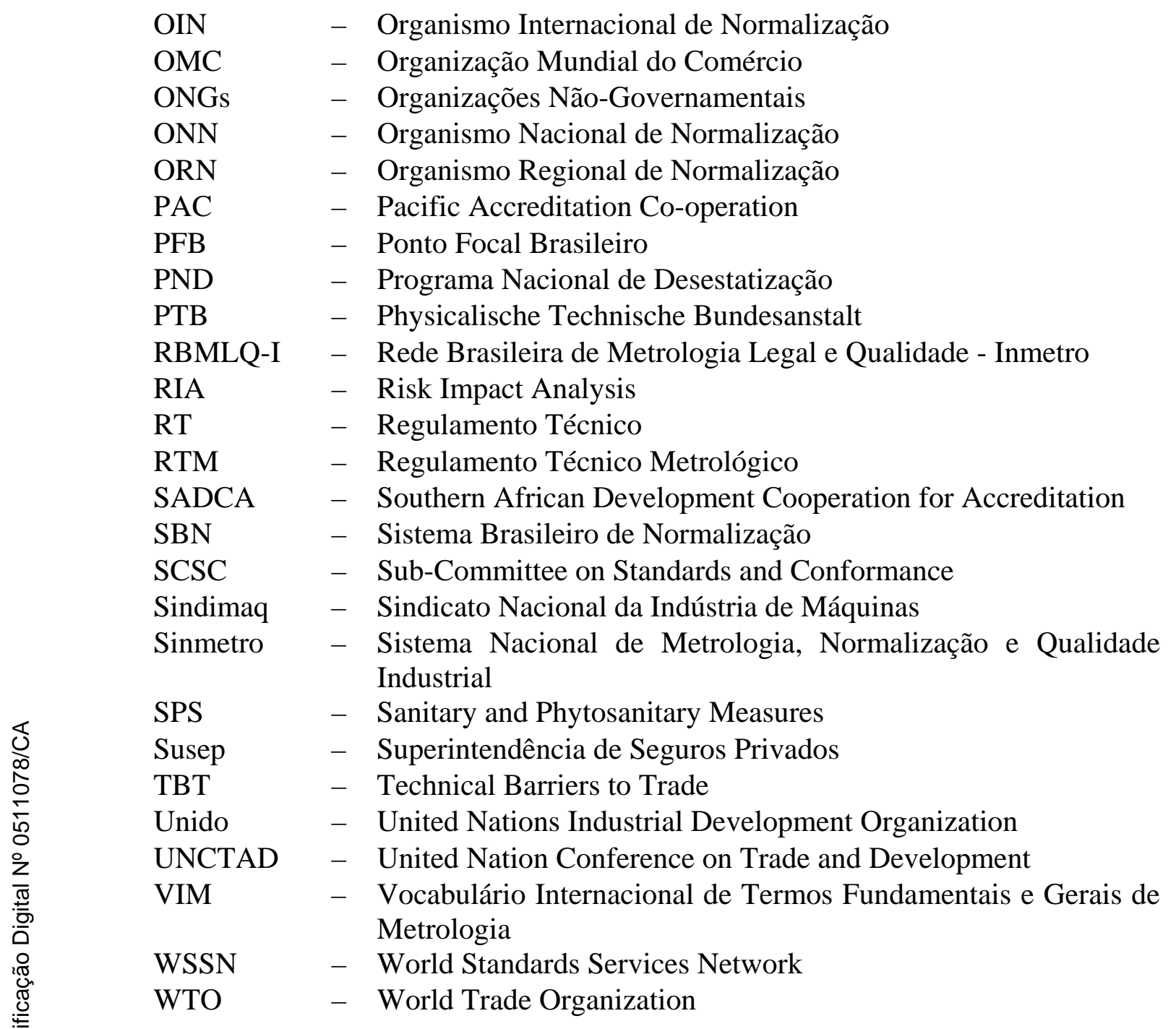




\section{Páginas web de interesse}

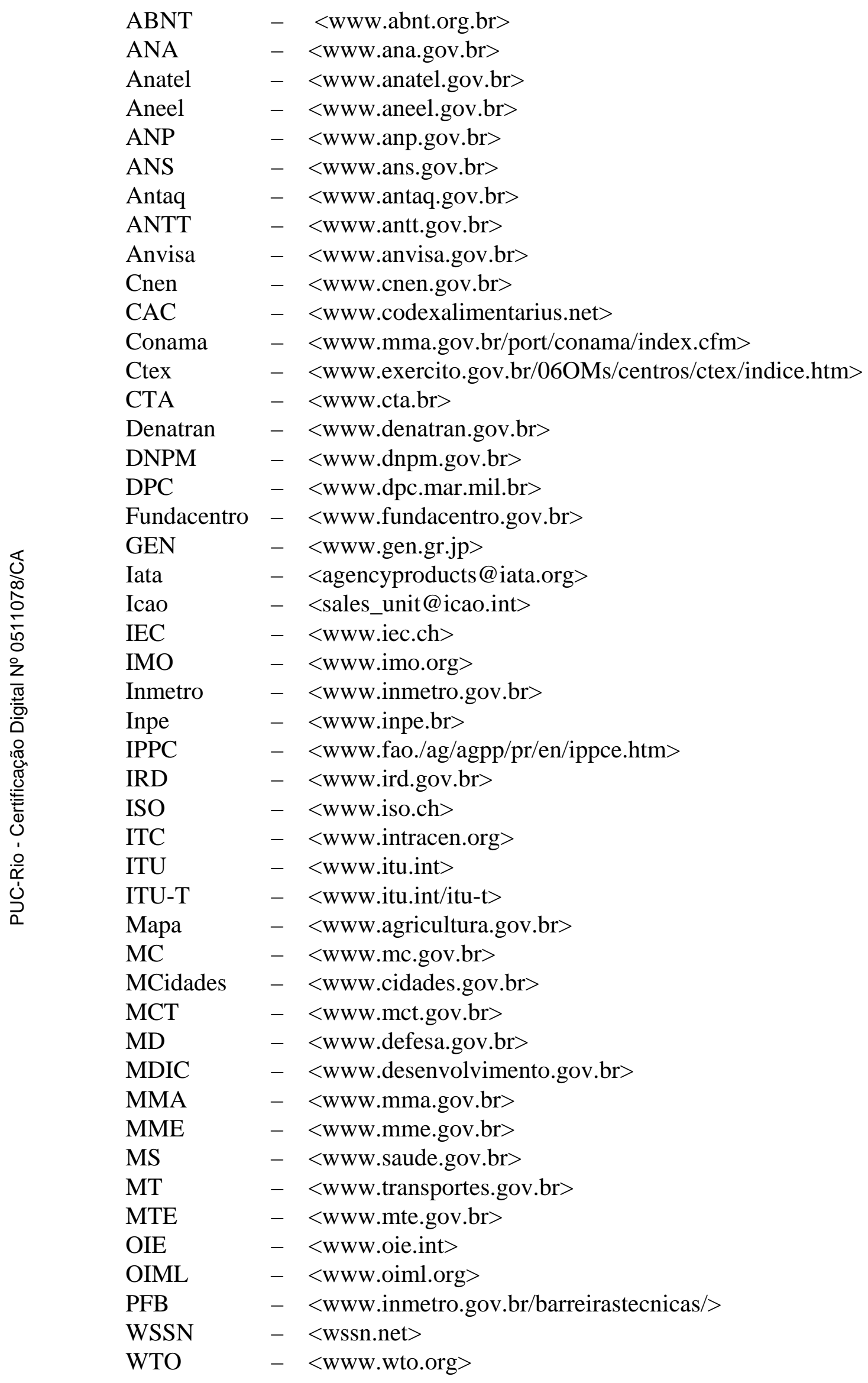

